\title{
Assistência prestada em serviços de saúde à população LGBTQIA+
}

\author{
Assistance in health services to the LGBTQIA+ population
}

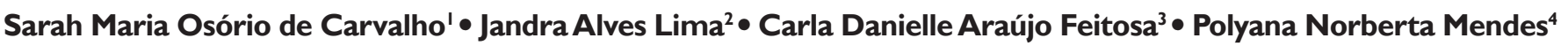

\begin{abstract}
RESUMO
Objetivo: analisar as produções científicas sobre a assistência em serviços de saúde à população LGBTQIA+. Metodologia: trata-se de uma revisão integrativa que incluiu artigos originais presentes nas seguintes bases de dados: Literatura Latino-americana e do Caribe (Lilacs) e Base de Dados de Enfermagem (Bdenf), acessadas via Biblioteca Virtual da Saúde (BVS);; a Medical Literature Analysis and Retrieval System Online (Medline) via Pubmed; e a Cumulative Index to Nursing and Allied Health Literature (CINAHL). Incluíram-se artigos primários publicados em qualquer idioma e que abordassem a temática investigada, sendo excluídos editoriais, teses, dissertações, artigos de revisão, bem como os duplicados. Resultado: a amostra foi composta por II artigos publicados em revistas nacionais e internacionais, organizados em três categorias temáticas: Barreiras na busca de cuidado de saúde;Acolhimento e o acesso aos serviços de saúde;Visão da população LGBTQIA+ perante o atendimento especializado. Conclusão: os estudos mostram que a assistência prestada a essa população ainda é permeada de estigma, preconceito por parte dos provedores de saúde, ocasionando a dificuldade de acesso aos serviços de saúde.
\end{abstract}

Palavras-chave: População LGBT; Assistência; Serviço de Saúde

\begin{abstract}
Objective:To analyze scientific productions about assistance in health services to the LGBTQIA+ population. Methodology: This is a bibliographical research of the integrative review type that included original articles present in the databases: Latin American and Caribbean Literature and the Nursing Database; a Medical Literature Analysis and Retrieval System Online accessed via PubMed and a Cumulative Index to Nursing and Allied Health Literature. Primary articles, published in any language and addressing the theme investigated and excluded from the editorial, theses, dissertations, review articles, as well as duplicates. Result:The sample consisted of II articles published in national and international journals, organized into 3 thematic categories: barriers in the search for health care, reception and accessed to health services, vision of the LGBTQIA+ population in the face of specialized care. Conclusion: Studies show that care provided for this population is still full of stigma, prejudice on the part of health providers causing the difficulty of access to health services.
\end{abstract}

Keywords: LGBT population; Assistance; Healthcare

I Acadêmica de Enfermagem, Centro Universitário Santo Agostinho (UNIFSA). Teresina-PI

2 Jandra Alves Lima.Acadêmica de Enfermagem. Centro Universitário Santo Agostinho (UNIFSA). Teresina-PI

3. Carla Danielle Araújo Feitosa. Enfermeira, graduada pela Universidade Federal do Piauí (20I5). Mestre em Enfermagem pelo Programa de Pós Graduação em Enfermagem da Universidade Federal do Piauí (20I8). Especialista em Enfermagem do Trabalho pela Faculdade Futura (20I9). Especialista em Enfermagem em Psiquiatria e Saúde Mental (2020). Membro do Grupo de Estudos e Pesquisas em Saúde Mental e Trabalho da Universidade Federal do Piauí. Desenvolve projetos vinculados à saúde mental, saúde do trabalhador e saúde do idoso.

4. Polyana Norberta Mendes. Centro Universitário Santo Agostinho (UNIFSA). Graduada em Enfermagem pela Universidade Federal do Piauí (20I5). Mestre em Enfermagem pelo Programa de Pós-Graduação em Enfermagem da Universidade Federal do Piauí (20I8). Docente do Curso de Graduação em Enfermagem do Centro Universitário Santo Agostinho (UNIFSA). Especialista em Enfermagem do Trabalho pela Faculdade Venda Nova do Imigrante (20I9). Membro do Grupo de Estudos sobre a Multidimensionalidade do Envelhecimento, Saúde e Enfermagem da Universidade Federal do Piauí. 


\section{INTRODUÇÃO}

Durante toda a história, verifica-se a existência de cidadãos e cidadãs que não correspondem ao papel social que lhes foi atribuído ao nascimento, vivenciando, assim, uma variedade de conflitos internos a respeito de si, principalmente por conviver em uma sociedade com padrões estabelecidos e preconceituosos. Sendo, portanto, uma das temáticas centrais de diversos debates no âmbito político, antropológico, biomédico e social ${ }^{(1,2)}$.

A identidade de gênero é uma classificação pessoal e social das pessoas em homens ou mulheres que pode ou não concordar com o gênero que lhes foi atribuído ao nascimento, sendo diferente do termo orientação sexual que está relacionado à atração que alguém sente por outro(s) indivíduo(s). É importante essa distinção de termos, uma vez que a população LGBTQIA+ é composta por uma heterogeneidade de pessoas, que são as Lésbicas, Bissexuais, Gays, Travestis e Transexuais, dentre outros ${ }^{(3)}$.

Essa população foi durante muitos anos privada dos seus direitos, da sua liberdade de expressão, de exercer a sua real identidade e alvo de discriminações, com destaque para a violência, em que estatísticas demonstram ser a população mais afetada. Já transcorreram vários anos desde a garantia dos seus direitos mediante constituição, políticas, portarias e leis, entretanto o cenário brasileiro ainda permanece praticamente o mesmo, não sendo uma situação diferente no campo da saúde ${ }^{(4)}$.

Ao considerar o direito à saúde garantido pela Constituição Federal e as leis orgânicas da saúde, instituiu-se por meio da Portaria $n^{\circ} 2.836$, de I de dezembro de 20I I, no Sistema Único de Saúde (SUS), a Política Nacional de Saúde Integral de Lésbicas, Gays, Bissexuais, Travestis e Transexuais. A implantação dessa normativa é uma vitória para a população, contudo ainda há grande discriminação por orientação sexual e por identidade de gênero que incide na determinação social da saúde, no processo de sofrimento e adoecimento decorrente do preconceito e do estigma social reservado às populações de Lésbicas, Gays, Bissexuais, Travestis e Transexuais ${ }^{(5,6)}$.

Todavia, a população LGBTQIA+ encontra dificuldades de operacionalização no que se refere ao acesso ao Sistema Único de Saúde (SUS) por permanecer com a ideia de que não segue o padrão heteronormativo dito pela sociedade. E aquilo que a torna "diferente", na maioria das vezes, pode ser usado contra ela nos serviços de saúde, logo termina tendo o seu direito de acesso à saúde violado, além de naturalizar um "não lugar" no SUS (2).

Nesse contexto, de lutas para ampliar e reafirmar os direitos da população LGBTQIA+, parte-se da questão norteadora: como se dá a assistência em saúde à po- pulação LGBTQIA+? Em vista disso, o presente estudo teve como objetivo analisar as produções científicas sobre a assistência prestada em serviços de saúde à população LGBTQIA+.

\section{METODOLOGIA}

Trata-se de uma revisão integrativa. A pesquisa foi norteada por protocolo construído pelos pesquisadores e seguiu a sequência de etapas a seguir: I) Elaboração da questão de pesquisa; 2) Determinação das bases de dados e seus critérios de inclusão e exclusão; 3 ) Extração de informações por meio de estudos primários; 4) Avaliação de estudos primários incluídos na revisão; 5) Análise e síntese dos resultados da revisão; 6) Apresentação da revisão integrativa ${ }^{(7)}$.

O Problema de pesquisa foi elaborado seguindo a estratégia: População Interesse Contexto (PICo). Obtevese a estrutura: P - população LGBTQIA+; I - assistência em saúde; Co - serviços de saúde. Dessa forma, elaborou-se o seguinte problema de pesquisa: como se dá a assistência em saúde à população LGBTQIA+?

O levantamento de dados foi realizado nos meses de julho e agosto de 2020, nas seguintes bases de dados: Medical Literature Analysis and Retrieval System Online (Medline) via Pubmed, Literatura Latino-americana e do Caribe (Lilacs) e a Base de Dados de Enfermagem (Bdenf), acessadas por meio da busca avançada na Biblioteca Virtual em Saúde (BVS), além da Cumulative Index to Nursing and Allied Health Literature (CINAHL) por ser uma base específica da Enfermagem.

Os critérios de inclusão foram artigos primários que apresentaram a assistência à população LGBTQUIA+ nos serviços de saúde como fenômeno de interesse e terem sido publicados em qualquer idioma. E os critérios de exclusão: editoriais, teses, dissertações, artigos de revisão integrativa, aqueles já selecionados na busca em outra base de dados e os que não responderam à questão da pesquisa.

Para a realização da busca nas bases de dados, selecionaram-se os Descritores em Ciência e Saúde (Decs), Medical Subject Headengs (MeSH), assuntos CINAHL e as palavras-chaves que são os descritores não controlados, definidos como sinônimos dos controlados. Os termos Decs, MeSh e as palavras-chaves foram utilizadas na Biblioteca Virtual da Saúde. $\mathrm{Na}$ Mediline via Pubmed, apenas os descritores MeSH. E a busca na CINAHL, os assuntos CINAHL. Para delimitar os termos de busca com mais de dois radicais utilizaram-se as aspas, visto que indicam a expressão exata para a pesquisa dos termos. Os termos foram combinados por meio dos operadores booleandos OR e AND, conforme Quadro I. 
QUADRO 1 - Expressão de busca nas bases de dados para seleção dos estudos primários. Teresina, PI,

Brasil, 2020.

\begin{tabular}{|c|c|}
\hline BASES DE DADOS & EXPRESSÃO DE BUSCA \\
\hline LILACS e Bdenf via BVS & 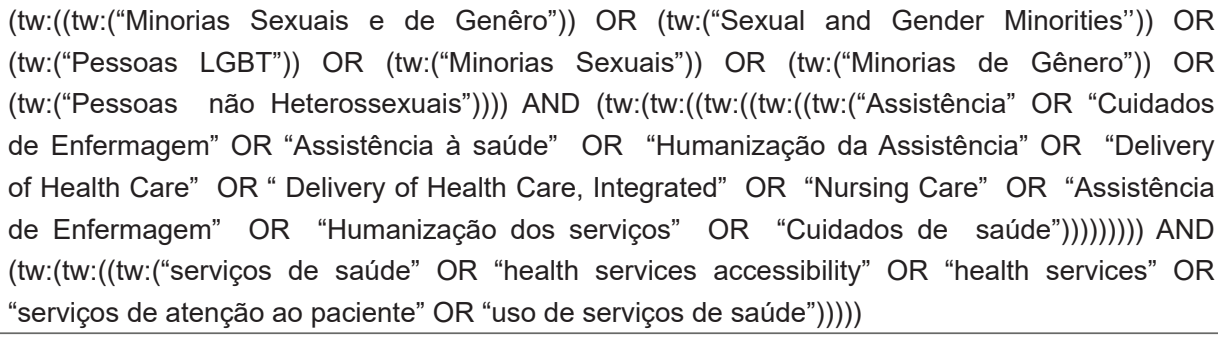 \\
\hline Medline via Pubmed & $\begin{array}{l}\text { ("Sexual and Gender Minorities"[MeSH Terms] AND (("Delivery of Health Care"[MeSH Terms] OR } \\
\text { "delivery of health care, integrated"[MeSH Terms]) OR "Nursing Care"[MeSH Terms])) AND ("Health } \\
\text { Services Accessibility"[MeSH Terms] OR "Health Services"[MeSH Terms]) }\end{array}$ \\
\hline CINAHL & $\begin{array}{l}\text { ( (MH "Gender Identity") OR (MH "Sexual Identity")) AND ( (MH "Health Care Delivery") OR } \\
\text { (MH "Health Care Delivery, Integrated") OR (MH "Nursing Care") ) AND ( (MH "Health Services } \\
\text { Accessibility") OR (MH "Health Services")) }\end{array}$ \\
\hline
\end{tabular}

Os termos Decs utilizados foram: Minorias sexuais e de gênero; Assistência; Cuidados de Enfermagem; Assistência a Saúde; Serviços de Saúde. Os termos MeSH: Sexual and Gender Minorities; Delivery of Health Care; Delivery of Health Care, Integrated; Nursing Care; Health Services Accessibility; Health Services. As Palavraschaves: Pessoas LGBT; Minorias Sexuais; Minorias de Gênero; Pessoas Não Heterossexuais; Assistência de Enfermagem; Cuidados de Saúde; Serviços de Atenção ao Paciente; Uso de Serviços de Saúde. E os assuntos CINAHL: Gender Identity; Sexual Identity;Health Care Delivery; Health Care Delivery, Integrated; Nursing Care; Health Services Accessibility; Health Services.

Realizou-se a busca por dois pesquisadores independentes, de forma simultânea, conforme protocolo, bem como a sequência dos descritores e os seus respectivos cruzamentos nas bases de dados a serem utilizadas; em seguida, compararam-se os dados obtidos por ambos os pesquisadores.

Os artigos da amostra foram selecionados por meio da sequência: leitura de título, leitura de resumo e leitura do texto integral. Nos casos em que ocorreram desacordos, houve discussão entre os dois avaliadores e análise por um terceiro para alcançar um consenso.

Para a coleta de informações pertinentes ao estudo, elaborou-se um instrumento de coleta de dados. As informações extraídas dos textos foram título, ano de publicação, região/instituição de vinculação dos autores, local de pesquisa, periódico da publicação, objetivo do estudo, nível de evidência, abordagem metodológica, população do estudo, assistência à saúde prestada, principais resultados dos estudos, forma de rastreio (instrumento de pesquisa), referencial teórico utilizado e o desfecho/conclusão.

Quanto ao nível de evidência, observou-se a seguinte classificação: nível I- metanálise de estudos controlados e randomizados; nível II- estudo experimental; nível III- es- tudo quase experimental; nível IV- estudo descritivo/não experimental ou abordagem qualitativa; nívelV- relato de caso ou experiência; nível VI- consenso e opinião de especialistas ${ }^{(8)}$.

Identificaram-se 975 publicações e após a aplicação dos critérios de inclusão e exclusão em cada base de dado, obteve-se uma amostra de II artigos para elaboração dos resultados, conforme apresentado na Figura I.

A análise crítica e a síntese qualitativa dos estudos selecionados foram realizadas de forma descritiva a partir da construção das categorias temáticas.

A pesquisa do tipo revisão integrativa não necessita de apreciação do Comitê de Ética, no entanto, reafirmase a garantia dos preceitos éticos e legais nas citações dos autores analisados para a obtenção dos resultados do presente estudo.

\section{RESULTADOS}

Selecionaram-se II artigos, sendo um $(9,09 \%)$ na Cinahl, um $(9,09 \%)$ na Lilacs, oito $(72,73 \%)$ na Medline e um $(9,09 \%)$ na Bdenf. Destes, destacam-se dois (18,18\%) em periódicos da enfermagem, cinco $(45,45 \%)$ em revistas interdisciplinares, três $(27,27 \%)$ em revistas e jornais que abordam a saúde coletiva e um $(9,09 \% \%)$ em revistas específicas que têm como área de interesse aspectos multifacetados da sexualidade e de gênero.

Após a análise dos artigos selecionados nesta revisão identificou-se que cinco $(45,46 \%)$ foram publicados no ano de 2018 , três $(27,27 \%)$ em 2017 , dois $(18,18 \%)$ em 2019 e um $(9,09 \%)$ em 2016 . Das publicações, destacam-se os trabalhos internacionais com nove $(81,82 \%)$ artigos e apenas dois $(18,18 \%)$ nacionais. Dos internacionais, quatro foram desenvolvidos nos Estados Unidos (36,37\%) e um em cada:Turquia, Canadá, Etiópia, Alemanha e África do Sul $(9,09 \%)$. Dos publicados nacionalmente, uma $(9,09 \%)$ pesquisa foi conduzida na capital do estado do Piauí e uma $(9,09 \%)$ no estado da Paraíba. 


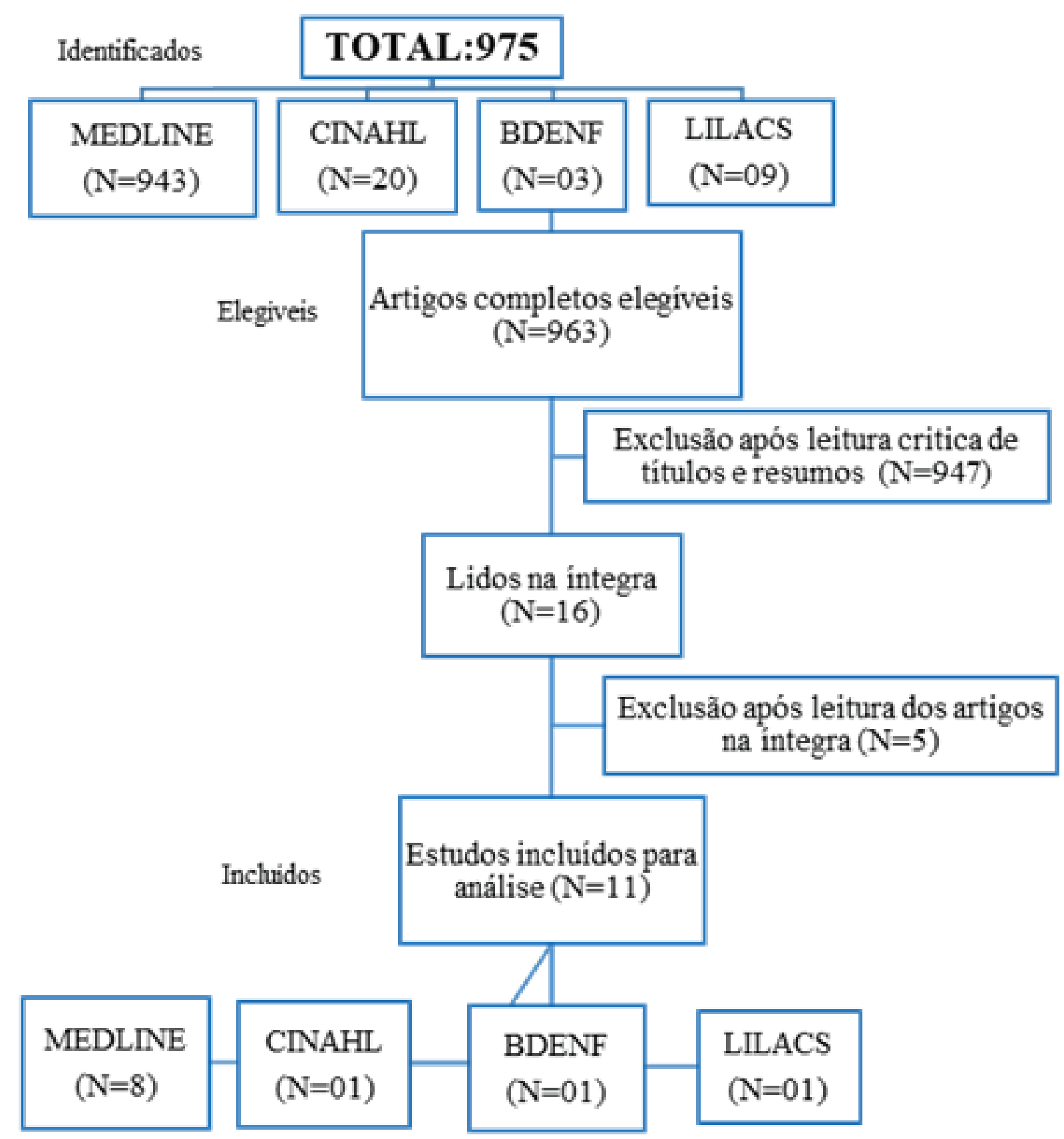

FIGURA 1 - Fluxograma de seleção dos estudos primários. Teresina, PI, Brasil, 2020.

No que tange à abordagem metodológica dos estudos, nove $(81,82 \%)$ são qualitativos e dois $(18,18 \%)$ estudos mistos.As estratégias utilizadas pelos pesquisadores para coleta de dados foram, em quatro (36,37\%), questionários e entrevistas, três $(27,27 \%)$ apenas questionário, um $(9,09 \%)$ somente entrevistas, um $(9,09 \%)$ grupos focais e um $(9,09 \%)$ questionários com grupos focais $(9,09 \%)$. Nenhum dos estudos primários fundamentou suas pesquisas em teorias. Quanto ao nível de evidência, dez $(90,91 \%)$ contemplaram o nível de evidência IV, que é estudo descritivo/ não experimental ou abordagem qualitativa, e um $(9,09 \%)$ o nível de evidência $V$, que se trata de relato de caso ou experiência.

No que diz respeito ao tipo de assistência à saúde prestada, estão a atenção primária à saúde com quatro
(36,36\%), a assistência oncológica com um (9,09\%) e os artigos que abordavam os vários serviços de saúde de maneira genérica com seis $(54,55 \%)$. Em relação às temáticas investigadas, têm-se as razões para não divulgação da identidade de gênero de jovens adultos LGBTQIA+, em um (9,09\%) artigo, as barreiras percebidas para busca dos cuidados com a saúde, em um $(9,09 \%)$, barreiras percebidas para atenção primária à saúde para a população LGBTQIA+, em três $(27,27 \%)$, fatores que determinam o comportamento de busca de cuidados de saúde, em um $(9,09 \%)$, estruturas de saúde, medidas de prevenção e diagnóstico, em um $(9,09 \%)$, e as experiências dos usuários LGBTQIA+ com os serviços de saúde, em três $(27,27 \%)$. Houve ainda um $(9,09 \%)$ dos estudos 
primários que analisou a percepção dos profissionais sobre a assistência e o acesso da população LGBTQIA+ aos serviços de saúde. Em relação à Política Nacional de Saúde Integral de Lésbicas, Gays, Bissexuais, Travestis e Transexuais, constatou- se que a mesma é abordada nas produções nacionais de forma pontual.

No que se refere à população em estudo, dez $(90,9 \%)$ foram com a população LGBTQIA+ e um $(9,09 \%)$ estudo foi realizado com profissionais da saúde a fim de conhecer a visão destes sobre a assistência a população LGBTQIA+. Dos dez estudos realizados com a população LGBTQIA+, sete $(70 \%)$ descreveram tanto a identidade de gênero quanto a orientação sexual, um ( $10 \%)$ apenas a identidade de gênero e dois $(20 \%)$ a orientação sexual dos participantes. Da população investigada sobre a identidade de gênero nesses artigos, a mulher fez parte dessa população em oito ( $80 \%$ ) artigos, homens em oito (80\%), mulher transexual em seis $(60 \%)$, homem transexual também em seis $(60 \%)$ e apenas um (10\%) incluiu o gênero não binário. No que diz respeito à orientação sexual, os gays foram investigados em oito $(80 \%)$, as lésbicas em nove ( $90 \%)$, os bissexuais foram abordados em cinco (50\%) artigos, os heterossexuais em cinco (50\%), os homossexuais em dois (20\%) e os assexuais e pansexuais foram pesquisados em três $(30 \%)$ dos artigos.

Os estudos foram divididos em três categorias, de acordo com o tipo de assistência à saúde prestada, sendo a categoria I Barreiras na busca de cuidados de saúde, categoria 2 Acolhimento e o acesso aos serviços de saúde e a categoria 3 Visão da população LGBTQIA+ perante o atendimento especializado. Na Figura 3 consta autor/ ano, objetivo, categoria e desfecho dos estudo primários.

QUADRO 2 - Síntese dos artigos da revisão segundo objetivo do estudo, categoria e desfecho. Teresina, PI, Brasil, 2020.

\begin{tabular}{|c|c|c|c|}
\hline CATEGORIA & AUTOR/ANO & OBJETIVO & DESFECHO \\
\hline Categoria 1 & $\begin{array}{c}\text { Getnet Tadele and } \\
\text { Woldekidan Kifle } \\
\text { Wolde/ } 2019^{(9)}\end{array}$ & $\begin{array}{l}\text { Examinar os fatores de intersecção que } \\
\text { determinam o comportamento de busca } \\
\text { de cuidados de saúde e a utilização de } \\
\text { serviços de saúde entre Lésbicas, Gays e } \\
\text { Bissexuais (LGB) na Etiópia. }\end{array}$ & $\begin{array}{l}\text { Percebeu-se que a homofobia, criminalização da } \\
\text { homossexualidade e heteronormatividade dos } \\
\text { serviços de saúde se cruzam com a oposição } \\
\text { social LGB, resultando em heterogeneidade de } \\
\text { risco, diversidade de saúde sexual e mental, } \\
\text { necessidades e diferença nos mecanismos de } \\
\text { enfrentamento. }\end{array}$ \\
\hline Categoria 1 & $\begin{array}{l}\text { Rubab I. Qureshi, } \\
\text { MD, PhD a, Peijia } \\
\text { Zha, PhD a, } \\
\text { Suzanne Kim et al } \\
\text { / } 2017^{(10)}\end{array}$ & $\begin{array}{l}\text { Explorar as barreiras percebidas para } \\
\text { buscar cuidados de saúde e a utilização } \\
\text { de cuidados de saúde entre lésbicas, gays, } \\
\text { bissexuais e transgêneros (LGBT) em Nova } \\
\text { Jersey. }\end{array}$ & $\begin{array}{l}\text { Em meios às barreiras encontradas nos } \\
\text { relatos dos pacientes, é nítido que há uma real } \\
\text { necessidade de um serviço de saúde mais } \\
\text { amplo, acessível e com foco no treinamento de } \\
\text { profissionais da saúde na sua diversidade de } \\
\text { especialidades. }\end{array}$ \\
\hline Categoria 1 & $\begin{array}{c}\text { Jacqueline } \\
\text { Gahagan and } \\
\text { Montse Subirana- } \\
\text { Malaret /2018 } \\
\text { (11) }\end{array}$ & $\begin{array}{l}\text { Explorar as principais preocupações com } \\
\text { a saúde, vias e barreiras percebidas para } \\
\text { atenção primária à saúde para populações } \\
\text { LGBTQ na perspectiva de uma amostra } \\
\text { da comunidade LGBTQ, membros e } \\
\text { prestadores de cuidados de saúde. }\end{array}$ & $\begin{array}{l}\text { É notório que ainda há uma grande necessidade } \\
\text { de executar a equidade e a igualdade no } \\
\text { ambiente da atenção primária. Dessa forma, } \\
\text { sugerem que os caminhos para a saúde primária } \\
\text { para população LGBTQ podem ser melhorados } \\
\text { abordando os problemas no nível micro do } \\
\text { indivíduo, ou seja, com foco nas barreiras } \\
\text { relatadas pelos pacientes/clientes. }\end{array}$ \\
\hline Categoria 1 & $\begin{array}{l}\text { Ute Lampalzer, } \\
\text { Pio Behrendt, } \\
\text { Arne Dekker, Peer } \\
\text { Brinken and Timo } \\
\text { O. Nieder/ } 2019 \\
\text { (12) }\end{array}$ & $\begin{array}{l}\text { Investigar as estruturas de saúde, medidas } \\
\text { de prevenção e diagnóstico, bem como } \\
\text { procedimentos de tratamento que os } \\
\text { indivíduos LGBTI precisam para receber } \\
\text { cuidados de saúde adequados centrados no } \\
\text { paciente e promoção da saúde. }\end{array}$ & $\begin{array}{l}\text { Constatou-se que os profissionais de saúde } \\
\text { precisarão adotar um melhor entendimento de } \\
\text { habilidades físicas específicas e consciência. } \\
\text { Tal atitude requer conhecimento básico sobre } \\
\text { questões de saúde relacionadas com LGBTI e } \\
\text { conhecimentos específicos sobre serviços de } \\
\text { saúde suficientes para cada uma das minorias no } \\
\text { contexto de sexo, orientação sexual e identidade } \\
\text { de gênero. }\end{array}$ \\
\hline
\end{tabular}




\begin{tabular}{|c|c|c|c|}
\hline Categoria 2 & $\begin{array}{l}\text { Geane Silva } \\
\text { Oliveira et al / } \\
\qquad 2018^{(13)}\end{array}$ & $\begin{array}{l}\text { Analisar, sob a ótica de profissionais da } \\
\text { Equipe Saúde da Família, o acesso de } \\
\text { lésbicas, gays, bissexuais e travestis/ } \\
\text { transexuais às Unidades Básicas de Saúde } \\
\text { da Família. }\end{array}$ & $\begin{array}{l}\text { Concluiu-se que os profissionais reconhecem } \\
\text { realmente a necessidade da equidade, o } \\
\text { acolhimento e a humanização do atendimento } \\
\text { no ambiente da atenção básica. Entretanto, } \\
\text { observou-se que os valores sociais e culturais } \\
\text { influenciam diretamente nas atitudes dos } \\
\text { profissionais. }\end{array}$ \\
\hline Categoria 2 & $\begin{array}{l}\text { Kinton Rossman, } \\
\text { Ph.D. Paul } \\
\text { Salamanca } \\
\text { and Kathryn } \\
\text { Macapagal, } \\
\text { Ph.D.Q / } 2017^{(14)}\end{array}$ & $\begin{array}{l}\text { Examinar as razões para a não divulgação } \\
\text { da identidade de jovens adultos LGBTQ } \\
\text { a prestadores de serviços médicos, bem } \\
\text { como experiências com prestadores após a } \\
\text { divulgação de identidade. }\end{array}$ & $\begin{array}{l}\text { Constatou-se um deficit no entendimento sobre } \\
\text { as questões LGBTQ por parte dos provedores, } \\
\text { principalmente relacionada à interação do } \\
\text { provedor/ paciente, sugerindo, dessa forma, } \\
\text { provedores de saúde mais treinados. }\end{array}$ \\
\hline Categoria 2 & $\begin{array}{l}\text { Barbara K. } \\
\text { Snyder, MD1, Gail } \\
\text { D. Burack, PhD2, } \\
\text { e Anna Petrova, } \\
\text { MD, PhD, MPH2/ } \\
2016{ }^{(15)}\end{array}$ & $\begin{array}{l}\text { Aprender sobre as experiências de jovens } \\
\text { LGBTQ com seus médicos da atenção } \\
\text { primária e identificar áreas para melhorias. }\end{array}$ & $\begin{array}{l}\text { Diante das experiências negativas relatadas } \\
\text { nos grupos focais, pode-se observar que os } \\
\text { participantes não se sentem nada confortáveis a } \\
\text { procurar um serviço de saúde. Portanto, para um } \\
\text { melhor acolhimento e atendimento, os provedores } \\
\text { de atenção primária precisam aumentar seus } \\
\text { conhecimentos sobre a saúde LGBTQ. }\end{array}$ \\
\hline Categoria 2 & $\begin{array}{l}\text { Alex Muller/ } 2017 \\
(16)\end{array}$ & $\begin{array}{l}\text { Analisar experiências dos usuários LGBT de } \\
\text { serviços de saúde. }\end{array}$ & $\begin{array}{l}\text { Observou-se que as pessoas que se identificam } \\
\text { como LGBT enfrentam vários desafios ao acessar } \\
\text { a saúde pública na África do Sul, visto que a falta } \\
\text { geral de recursos no sistema de saúde público } \\
\text { da África do Sul leva à discriminação sistemática } \\
\text { contra pessoas de orientação sexual não } \\
\text { normativa e/ou identidades de gênero. }\end{array}$ \\
\hline Categoria 2 & $\begin{array}{l}\text { Breno de Oliveira } \\
\text { Ferreira, Jose } \\
\text { Ivo dos Santos } \\
\text { Pedrosa, Elaine } \\
\text { Ferreira do } \\
\text { Nascimento/ } \\
\text { 2017. (2) }\end{array}$ & $\begin{array}{l}\text { Apreender as dimensões do acesso e } \\
\text { da atenção integral na rede do Sistema } \\
\text { Único de Saúde (SU) na perspectiva da } \\
\text { diversidade de gênero. }\end{array}$ & $\begin{array}{l}\text { Conclui-se que há emergência no acesso às } \\
\text { redes de atenção à saúde integral da população } \\
\text { LGBT com o intuito de promover a inclusão em } \\
\text { seus diversos equipamentos sociais, promulgar o } \\
\text { respeito e facilitar práticas de equidades. }\end{array}$ \\
\hline Categoria 3 & $\begin{array}{l}\text { Ellen Carr/ } 2018 \\
\qquad(17)\end{array}$ & $\begin{array}{l}\text { Capturar as perspectivas de lésbicas, } \\
\text { gays, bissexuais e indivíduos transgêneros } \\
\text { (LGBT) com diagnóstico de tratamento para } \\
\text { câncer. }\end{array}$ & $\begin{array}{l}\text { Constatou-se que o cuidado com o paciente } \\
\text { LGBT é baseado na sensibilidade e consciência } \\
\text { às questões e preocupações LGBT. }\end{array}$ \\
\hline Categoria 3 & $\begin{array}{l}\text { Sukru Kelles, } \\
\text { Mustafa Volkan } \\
\text { Kavas e Neyyre } \\
\quad \text { Yasemin } \\
\text { Yalim/2018 }\end{array}$ & $\begin{array}{l}\text { Avaliar como os indivíduos LGBT refletem } \\
\text { sobre seus próprios corpos, sexualidade e } \\
\text { gênero. }\end{array}$ & $\begin{array}{l}\text { Concluiu-se que a abordagem profissional dos } \\
\text { médicos tem uma influência considerável nos } \\
\text { indivíduos LGBT+ para a utilização de serviços } \\
\text { de saúde. }\end{array}$ \\
\hline
\end{tabular}

\section{DISCUSSÃO}

\section{Barreiras na busca de cuidados de saúde}

A análise dos estudos selecionados para compor esta categoria indica que muitas barreiras estão presentes e são motivo para não buscar os cuidados de saúde.
Uma pesquisa realizada na Etiópia mostrou que apenas $35,5 \%$ dos entrevistados relataram que estão sempre motivados a buscar atendimento quando não estão se sentindo bem e uma proporção de 58 , 1 \% afirmou que só se sente motivada às vezes. $O$ motivo que os participan- 
tes citaram para a não procura é o medo de ser estigmatizado pelos seus provedores de saúde. Outras questões que também influenciaram esse comportamento de não busca foram a falta de dinheiro, referiram não haver médicos sensíveis e a distância da unidade de saúde, pois geralmente procuram cuidados em outras comunidades para não serem descobertos por familiares e amigos ${ }^{(9)}$.

Acrescenta-se, ainda, a má assistência prestada pelos profissional como uma das principais barreiras mencionadas, obtendo-se, assim, sempre os mesmos resultados, que são a recusa dos profissionais em atender os clientes pertencentes a essa população, a falta de conhecimento, treinamentos e competência cultural pelos provedores, 0 preconceito exposto na assistência, a mudança de comportamento dos profissionais quando o cliente expõe a orientação sexual, fazendo com que muitos nem revelem $\mathrm{e}$, inclusive, omitam as doenças relacionadas a sua sexualidade, além da falta de profissionais especializados para atender todos aqueles que fazem parte da população LGBTQIA+ $(9,10,11,12)$.

Houve ainda relato em dois dos artigos que compõem esta categoria sobre a falta de convivência da população nas unidades de saúde, pois não há um estímulo por parte dos serviços. Um exemplo bastante citado foi a organização da atenção primária à saúde, na qual possui o dia da mulher, hiperdia, puericultura, mas não há nenhum dia ou programa com foco na população nem educação em saúde que visem às situações que mais acometem essa população. Inclui-se também a falta de atenção psicossocial e psicoterapêutica, a longa espera pelos especialistas e a falta de um ambiente acolhedor ${ }^{(9,12)}$.

Nessa pespectiva de discriminação institucional, as assistências prestadas pelos profissionais de saúde deve estabelecer prioritariamente a empatia, de modo que possa obter uma melhor compreensão das necessidades exigidas, e tendo por finalidade suprir as expectativas dessa população com o desenvolvimento de ações e serviço de saúde destinados a atender, especificamente, as peculiaridades da população LGBTQIA+, tendo em vista também, em proporcionar um vínculo e um maior conhecimento aos profissionais da saúde, favorecendo a realização de uma prática eficaz com ênfase na ética profissional ${ }^{(19,20,21)}$

\section{Acolhimento e o acesso aos serviços de saúde}

Apontou-se, nesta categoria, a avaliação do acolhimento da população LGBTQIA+ em vários serviços de saúde.Tem-se o acolhimento como um método pensado e implementado a fim de colaborar com a qualificação dos sistemas de saúde, de maneira que possibilite ao usuário o acesso a um atendimento justo e pautado na integralidade, com o objetivo de escutar e atender as suas necessidades ${ }^{(22)}$.

A presença de generalização e desconhecimento das especificidades da população LGBTQIA+ por parte dos profissionais de saúde resulta na desigualdade no acesso e no acolhimento nos serviços de saúde. Sendo que o acesso aos serviços de saúde é um dos grandes desafios para a população LGBTQIA+, tanto no setor público como no privado ${ }^{(13)}$.

Estudos incluídos nesta categoria mencionam como uma preocupação das minorias sexuais a questão do sigilo médico, os comentários desnecessários entre profissionais de saúde sobre a sua identidade e orientação sexual. Nessa circunstância, muitos relatam a dificuldade no acolhimento e acesso ao divulgarem a sua identidade e orientação sexual, sendo que a maioria teve os seus cuidados negados e quando não sofrem microagressão e discriminações ${ }^{(15,23)}$.

Há uma insatisfação dessa população quanto à comunicação com a equipe médica durante as consultas, por conseguinte a necessidade de se discutir a respeito da sua saúde sexual, orientação sexual, comportamento e educação voltada às doenças sexualmente transmissíveis. Diante disso, muitos sugerem uma melhor comunicação ${ }^{(15)}$.

A discriminação, o desrespeito e as agressões verbais não advêm somente dos profissionais da saúde, mas igualmente da equipe gestora e administrativa do serviço de saúde, e até mesmo dos outros clientes, o que foi evidenciado em um dos estudos, em que a população investigada se tratava de profissionais da saúde. Entre os relatos, eles afirmam ter receio de atender esse público porque sentem medo de serem vistos pela sociedade ${ }^{(16)}$.

Os motivos que levam a procurarem os serviços de saúde estão voltados às consultas de emergência, à busca de preservativos e à realização de exames. Os participantes afirmaram que necessitam de serviço de saúde que promova um melhor acolhimento, vínculo e uma atenção maior no cuidado ${ }^{(13)}$.

Nessa perspectiva, esse público também ratifica o desejo de um acompanhamento específico, bem como de todas as áreas profissionais, ou seja, médicos, enfermeiros, técnicos de enfermagem, odontologista, entre outros, que tivessem a capacitação no acolhimento e tratamento desses pacientes nos serviços de saúde ${ }^{(13,24)}$.

\section{Visão da população LGBTQIA+ perante o atendi- mento especializado}

Nesta categoria, buscou-se examinar como a população LGBTQIA+ observa o atendimento especializado. No contexto do SUS, a atenção especializada está relacionada a um conjunto de procedimentos que envolvem alta tecnologia e alto custo, objetivando proporcionar à população acesso a serviços qualificados, integrando-os nos demais níveis de atenção à saúde, sendo tanto na atenção básica como na de média complexidade ${ }^{(25)}$.

Em uma investigação realizada com pacientes LGB- 
TQIA+ em uma clínica oncológica sobre suas experiências pessoais com relação ao atendimento feito por enfermeiros oncológicos, estes relataram experiências positivas com o acolhimento e a assistência desses profissionais de saúde. Nesse estudo, concluiu-se que, quando se trata de um atendimento especializado a pacientes LGBTQIA+ com câncer, esses profissionais de saúde se atentam ao cuidado e à atenção livre de qualquer estigma ou discriminação para com esse público (17).

Destaca-se, ainda, a importância do acolhimento, da sensibilidade e conscientização dos profissionais de enfermagem oncológicos, de maneira que possam se tornar referência de atendimento dos usuários LGBTQIA+ para outras áreas temáticas. Além disso, chama atenção dos pacientes LGBTQIA+ com câncer buscar conhecer os seus direitos nos serviços de saúde especializado (17).

No entanto, ao contrário do estudo anterior, há relatos de problemas de acesso e cuidado especializado, tais problemas são preconceito, discriminação, maus-tratos, marginalização e dificuldade de autoexpressão. Esses mesmos pacientes narraram que estão tendo que formular um discurso ao estarem com os profissionais de saúde, especialmente com os médicos ${ }^{(18)}$.

Além do mais, o mesmo estudo enfatiza o quanto é importante que as escolas médicas e outras instituições de ensino incluam informações necessárias nos seus históricos escolares sobre as necessidades das minorias sexuais nos serviços de saúde. Em vista disso, identificou-se que ainda é preciso melhorar o atendimento nos serviços de saúde, sobretudo o atendimento especializado, de tal modo que conscientize os profissionais de saúde para um atendimento mais empático ${ }^{(18)}$.

Os estudos sobre a asssistência à saúde da população LGBTQIA+ são divulgados principalmente em revistas interdisciplinares e de saúde coletiva, mas também em periódicos da enfermagem e em revistas específicas cuja área temática são as questões de gênero. As revistas ao abordarem a multifacetada questão da sexualidade e gênero mostram que em alguns países vem emergindo a necessidade de ampliar a discussão e a produção científica sobre essa temática. Os autores dos artigos analisados são das diversas áreas que integram a saúde, tornando-se um ponto positivo, já que as questões culturais enraizadas e o pouco conhecimento sobre a temática em questão estão estritamente relacionados aos problemas envolvidos com a má assistência nos serviços de saúde (II).

Constatou-se que os estudos selecionados foram realizados em diferentes países, tais como Turquia, Canadá, Etiópia, Alemanha e África do Sul, com destaque para os Estados Unidos e Brasil. Entretanto, sentiu-se a necessidade de mais publicações que incluam o objeto de estudo em países subdesenvolvidos, nos quais as disparidades sociais e o estigma são um grande obstáculo enfrentado diariamente por essa população. No Brasil, as publicações podem estar relacionadas com as altas taxas de crimes voltados para a homofobia, liderando durante vários anos o ranking de países que mais matam a população LGBTQIA+, sendo a divulgação científica uma das formas de agregar conhecimento e mudar a visão tanto dos profissionais quanto da sociedade contemporânea (26).

Em relação aos anos de publicações, as produções foram divulgadas entre os anos de 2016 e 2019 , tendo o ano de 2018 mais publicações. Trata-se de estudos recentes que descrevem o cenário atual, em todo o mundo, sobre a temática e que possuem como objetivo comum agregar conhecimento para garantir que essa população possua uma assistência à saúde de qualidade.

Quanto às metodologias utilizadas nos estudos, destacam-se os estudos qualitativos com uso de estratégias para a coleta de dados, entrevistas, questionários e grupos focais e um único relato de experiência, estando, pois, nos níveis de evidência IV e V, respectivamente. A classificação do nível de evidência divide os estudos em cinco níveis baseados no tipo de estudo, quanto maior a numeração menor será a força da evidência para recomendações clínicas. No entanto, esses métodos são relevantes, visto que permitem uma aproximação para conhecer a percepção, os sentimentos, o vivido dos entrevistados e assim identificar os problemas enfrentados no cotidiano. Estudos como esses possibilitam ampliar a visão sobre o público-alvo. No entanto, limitam a generalização dos resultados obtidos ${ }^{(8)}$.

Os estudos analisados foram divididos em três categorias temáticas. Logo, constatou-se a partir das categorias que existe resistência, medo e preconceito por parte dos profissionais da saúde, como também da gestão, em atender esse público. Os estudos apontam que as Instituições de Ensino e os próprios gestores dos serviços de saúde têm falhado na formação de profissionais sensíveis às demandas de saúde da população LGBTQIA+, sendo relatada uma lacuna no conhecimento acerca das necessidades dessa população, o que consequentemente dificulta $o$ acesso aos serviços de saúde. Dentre os relatos dos participantes, destaca-se a falta de compreensão dos provedores a respeito das questões de saúde que envolvem a população ${ }^{(14)}$.

No que se refere à organização da assistência à saúde, a maioria dos estudos abordou a assistência de maneira genérica; em seguida, destaca-se a atenção primária à saúde e, por fim, a assistência oncológica. Ao analisar os variados tipos de assistência, constatou-se que o acesso aos cuidados de saúde ainda enfrenta o preconceito, os estigmas, a falta de conhecimento dos profissionais, o que traz impactos negativos na assistência, principalmente no acolhimento da população LGBTQIA+ ${ }^{(14)}$.

Ainda, mostra-se distante a garantia de saúde como 
direito, um atendimento com qualidade integral, universal e equânime, mas vale destacar a importância de políticas específicas, uma vez que foi a partir delas que se trabalhou com a chamada inclusão. É preciso lembrar que a população LGBTQIA+ é ainda a mais distanciada dos serviços de saúde e isso ocorre especialmente por falta de mecanismos específicos que facilitem o acesso dessa população aos serviços ${ }^{(27)}$.

As produções nacionais citam a política nacional de saúde integral de Lésbicas, Gays, Bissexuais, Travestis e Transexuais no que diz respeito a sua importância e seu fortalecimento, como também a necessidade de capacitações para os profissionais da Estratégia Saúde da Família que ainda tem uma visão distorcida sobre a abordagem da sexualidade unicamente baseada em políticas e diretrizes específicas. A política é uma das maiores conquistas para essa população por proporcionar melhorias em vários âmbitos, principalmente nos serviços de saúde. No entanto, constatou-se que, nos estudos analisados, sentiu-se a necessidade de abordarem a mesma nos seus instrumentos de coleta, visto que é mais que necessário saber a visão da população sobre os seus direitos garantidos $(2,13,28)$.

Nesse caso, entende-se que mesmo com a existência de leis que proporcionam os direitos dessa população as literaturas enfatizam, ainda, a suma importância de um avanço no campo dos direitos sexuais e a quebra de paradigmas sociais e culturais que, na maioria das vezes, impedem aquela de buscar a assistência nos serviços de saúde ${ }^{(14)}$.

Aponta-se a indignação por parte da população LGBT$\mathrm{QIA}+$ no atendimento realizado, especialmente pela equipe médica. A maioria dos relatos apresentados demonstrou que esse público se sente intimidado quando está diante de um profissional médico por negar assistência, tendo em vista que a falta de conscientização e sensibilidade desses profissionais de saúde tem por consequência ações negativas, como preconceito e discriminação ${ }^{(15)}$.

Mesmo na presença de tantas políticas beneficiando à população LGBTQIA+, estes ainda precisam ser orientados corretamente quanto aos seus direitos e deveres dentro do Sistema Único de Saúde, além de tudo, mantendo uma postura reflexiva frente aos padrões heterossexuais estabelecidos na sociedade, colaborando para uma assistência humanizada e holística, prevenindo a descriminalização e buscando satisfazer as necessidades específicas dessa população, visto que tais orientações ainda são pontuais e desarticuladas o que demonstra fragilidade na formação desses provedores de saúde e na divulgação de informações para propria população ${ }^{(29,30)}$

$\mathrm{Na}$ terceira categoria, analisou-se a visão da popula- ção LGBTQIA+ sobre o atendimento especializado na oncologia. Os relatos foram de satisfação por parte dos pacientes oncológicos LGBTQIA+ que argumentaram serem bem atendidos pelos profissionais da enfermagem oncológica. Tais argumentos descrevem que esses profissionais garantem o conforto e a segurança, além de estimular a realização do tratamento em uma perspectiva mais positiva. Não obstante, esse público salienta que esses profissionais da enfermagem oncológica podem servir de modelo para outros profissionais da saúde, como também para outras especialidades da categoria $(17,18)$.

Entende-se, portanto, que há uma extrema necessidade de capacitação dos profissionais de saúde, bem como de todos que fazem parte dos serviços de saúde, com relação ao acolhimento, acesso e assistência prestada à população LGBTQIA+. Em vista disso, a fiscalização desses profissionais da saúde perante os pacientes LGBTQIA+ seria um avanço para a ruptura dos paradigmas existentes, o que impossibilita a busca pelo atendimento de saúde ${ }^{(13)}$.

Aponta-se, como limitação desta revisão a prevalência de estudos primários com delineamento transversal, impossibilitando a verificação de relações de causa e efeito e a generalização dos resultados sobre a assistência a população LGBTQIA+ prestada nos serviços de saúde.

\section{CONCLUSÃO}

A assistência à saúde da população LGBTQIA+ relatada nos estudos avaliados indica a cofluência de preconceito, estigmas e discriminações que estão presentes desde $o$ acolhimento até os cuidados diretos e indiretos fornecidos pelos provedores de saúde.

A Política Nacional de Saúde Integral de Lésbicas, Gays, Bissexuais, Travestis e Transexuais possui objetivos bem estruturados, entretanto a mesma não é referencial para as discussões das produções científicas nacionais, o que possivelmente demonstra a sua, ainda, incipiente aplicabilidade nos serviços de saúde, sendo também pouco divulgada.

Entre as principais barreiras enfrentadas estão a dificuldade de acesso, ocasionada em parte pelo pouco conhecimento dos profissionais sobre as necessidades de saúde da população LGBTQIA+, e a pouca estrutura fornecida a essa população. Concluiu-se que, no cenário atual, a população LGBTQIA+ ainda padece com dificuldades de acesso aos serviços de saúde. Dessa forma, sugere-se que a Enfermagem inclua na graduação discussões e ações que trabalhem a inclusão, a equidade e a igualdade em torno desse grupo, bem como que os gestores dos serviços de saúde invistam em qualificação para os profissionais. 


\section{REFERÊNCIAS}

I - Pacheco, R.A.S.; Pacheco, I.S. Direito, violências e sexualidade: a transexualidade em um contexto de direitos. Sócio-Jurídicos [ Internet]. 2016 [acesso em 23 mar 2020]; I8(2): 203-228. Disponível em: http:// www.scielo.org.co/scielo.php?script=sci_abstract\&pi$\mathrm{d}=\mathrm{S} 0$ I 24-057920 I 6000200007

2 - Ferreira, B.O.; Pedrosa, J.I.S.; Nascimento, E.F. Diversidade de gênero e acesso ao sistema único de saúde. Rev brasileira em promoção da saúde [Internet]. 2017 [acesso em 25 mar 2020]; 3 I(I). Disponível em: https://periodicos.unifor. $\mathrm{br} / \mathrm{RBPS} / \mathrm{article/view/6726.}$

3 - Ministério da Saúde (BR), Secretaria de vigilância em saúde. Departamento de doenças de condições crônicas e infecções sexualmente transmissíveis. Protocolo Clínico e Diretrizes Terapêuticas para Atenção Integral às Pessoas com Infecções Sexualmente Transmissíveis (IST). Brasília (DF); 2019. [acesso em 21 mar 2020]. Disponível em: http://www. aids.gov.br/pt-br/pub/2015/protocolo-clinico-e-diretrizesterapeuticas-para-atencao-integral-pessoas-com-infeccoes.

4 - Carvalho, L.S.; Philippi, M.M. Percepção de lésbicas, gays e bissexuais em relação aos serviços de saúde. Rev Ciências da saúde [Internet]. 2013 [acesso em 22 de mar 2020]; I I(2): 83-92. Disponível em: https://www.publicacoesacademicas.uniceub.br/cienciasaude/article/view//837/2286.

5 - Ministério da Saúde (BR). Portaria n².836, de I de Dezembro de 20II. Institui a Política Nacional de Saúde Integral de Lésbicas, Gays, Bissexuais, Travestis e Transexuais. Diário Oficial. I dez 20I I. [acesso em 21 mar 2020]. Disponível em: http://bvsms.saude.gov.br/bvs/saudelegis/gm/20I I/ prt2836_0l_12_20II.html.

6 - Da Silva,T.A et al. Movimento lgbt, políticas públicas e saúde. Rev de Psicopedagogia, Psicologia escolar e Educação [Internet]. 2018 [acesso em 25 mar 2020]; 2 I (I): I9I-208.

Disponível em: https://www.periodicos.ufam.edu.br/amazonica/article/view/47I5.

7 - Mendes, K. D. S. ; Silveira, R. C. C. P.; Galvão, C. M. Revisão integrativa: método de pesquisa para a incorporação de evidências na saúde e na enfermagem integrative literature. Florianópolis:Texto \& Contexto Enferm; 2008. p. 758-764.

8 - Melnyk, B.M; Fineout-Overholt, H. Evidence-based practice in nursing and healthcare: a guide to best practice. Philadelphia: Lippincott Williams \& Wilkins;2005. P. 3-24.

9 -Tadele, G;Amde,W.K. Health needs, health care seeking behaviour, and utilization of health services among lesbians, gays and bisexuals in Addis Ababa, Ethiopia. International journal for equity in health [Internet]. 2019 [acesso em 21 agost 2020]; I8(I):86. Disponível em: https://www.ncbi.nlm.nih.gov/ pmc/articles/PMC6560764/.

10 - Quereshi, R. I; Zha, P; Kim, S; Hindin, P; Naqvi, Z; Holly, C; Dubbs, W; Ritch, w. Health care needs and care utilization among lesbian, gay, bisexual, and transgender populations in new jersey.J homosex [Internet]. 2018 [acesso em 21 agost
2020]; 65(2): I67-I80. Disponível em: https://pubmed.ncbi. nlm.nih.gov/2848I7I8/.

I I- Gahagan, J; Malaret, S.M. Improving pathways to primary health care among LGBT populations and health care providers: key findings from Nova Scotia, Canada. International journal for equity in health [Internet]. 2018 [acesso em 21 agost 2020]; 17: 76. Disponível em: https://www.ncbi.nlm.nih.gov/ pmc/articles/PMC5998559/.

12 - Lampalzer, U; Behrendt, P; Dekker, A; Briken, P; Nieder, T.O. The needs of LGBTI people regarding health care structures, prevention measures and diagnostic and treatment procedures: A qualitative study in german metropolis. International Journal of [Internet]. 2019 [acesso em 21 agost 2020]; I6(19): 3547. Disponível em: https://www.ncbi.nlm.nih.gov/pmc/articles/PMC680 I863/pdf/ijerph-16-03547.pdf

13 - Oliveira, G.S; Nogueira, J.A; Costa,G.P.O; Fonseca, R.L.S; Medeiros, M; Oliveira, T;Almeida, S.A. Serviços de saúde para lésbicas, gays, bissexuais e travestis/transexuais. Revista de Enfermagem UFPE on line [Internet]. 2018 [acesso em 2I agost 2020]; 12(I0): 2598- 609. Disponível em: https://periodicos. ufpe.br/revistas/revistaenfermagem/article/view/2370I4.

I4 - Rossman, K; Salamanca, P; Macapagal, K. "The doctor saind I dindn't look gay":Young adults'experiences of disclosure and non-disclosure of LGBTQ identity to healthcare providers. J homosex [Internet]; 2018 [acesso em 02 set 2020]; 64(10): 1390-|410. Disponível: https://www.ncbi.nlm.nih. gov/pmc/articles/PMC5772907/.

15 - Snyder, B. K; Burack, G.D; Petrova, A. LGBTQ Youth's perceptions of primary care. Clinical pedriatrics [Internet]. 2016 [acesso em 03 set 2020]; 56(5): 443-450. Disponível em: https://journals.sagepub.com/doi//0.I I77/00099228/6673306? url_ver=Z39.88-2003\&rfr_id=ori:rid:crossref.org\&rfr_dat=cr_pub\%20\%200pubmed.

16 - Muller, A. Scrambling for access: availability, accesibility, acceptabolity anda quality of healthcare for lesbian, gay, bisexual and transgender people in south Africa. BMC int healt hum rights [Internet]. 2017 [acesso em 03 set 2020]; I7(I): 16. Disponível em: https://www.ncbi.nlm.nih.gov/pmc/articles/ PMC5450393/.

17- Carr, E. The personal experience of LGBT patients with cancer. Seminars in ncology nursing [Internet]. 2018 [acesso em 02 set 2020]; 34(I): 72-79. Disponível em: https://www.sciencedirect.com/science/article/abs/pii/ S0749208II730I 237.

18 - Keles, S; Kavas, M.V; Yalim, N.Y. LGBT + individuals' perceptions of healthcare services in turkey:A cross- sectional qualitative study. Journal of biothical inquery[Internet];2018 [acesso em 02 set 2020]; I5: 497-509. Disponível em: https://link.springer.com/article/ I0.1007/s I I673-0 I8-9874-5.

19 - Popadiuk, G.S.; Oliveira, D.C; Signorelli, M.C. A Política Nacional de Saúde Integral de Lésbicas, Gays, Bissexuais e Trans-gêneros (LGBT) e o acesso ao Processo Transexualizador no Sistema Único de Saúde (SUS). Ciências \& Saúde 
Coletiva [Internet]. 2017 [acesso em 25 set 2020]; 22(5): I509-I520. Disponível em: http://www.scielo.br/scielo. php?pid=S I $4|38| 23201700250 \mid$ 509\&script=sci_abstract\&ting=pt.

20 - Costa LD, Barros AD, Prado EAJ, Sousa MF, Cavadinha ET, Mendonça AVM. Competência Cultural e Atenção à Saúde da população de lésbicas, gays, bissexuais travestis e transexuais (LGBT) Tempus, actas de saúde colet. 2017[acesso em 25 set 2020]; I I ( ): I05- I 19. Disponível em: http://www. tempusactas.unb.br/index.php/tempus/article/view/ 895.

21 - Cavalcanti AC. Acolhimento nos Serviços de Saúde à População LGBT: Uma Revisão Integrativa. Rev do Congresso Brasileiro de Ciências da Saúde. 2016;I(I):I- 9. Disponível em: https://editorarealize.com.br/revistas/ conbracis/trabalhos/TRABALHO_EV055_MDI_SA4_ ID365_30052016231804.pdf.

22 - Santos, L.E.S;Fontes,W.S.F.; Oliveira,A.K.S.;Lima,L.H.O.,Silva, A.R.V.O acesso ao Sistema Único de Saúde na percepção de homossexuais masculinos. Rev Brasileira de Enfermagem [Internet]. 2020 [acesso em 21 agost 2020]; 73(30). Disponível em:https://www.scielo.br/scielo.php?script=sci_arttext\&pi$d=S 0034-7 \mid 672020000200$ | $86 \&$ Ing=pt\&nrm=iso\&tlng=pt.

23 - Almeida, F.A; A dificuldade de acesso de usuários (as) do processo transexualizador aos serviços de hormonioterapia. Congresso Brasileiro de Assistentes Sociais [Internet]. 2019[acesso em 25 set 2020]; 23(I). Disponível em: http://www.scielo.br/scielo.php?script=sci_arttext\&pi$\mathrm{d}=\mathrm{S}|4|$ |4-328320 $9000 \mid 00268$.

24 - Pereira, E.O.Acesso e Qualidade da Atenção à Saúde para a População LGBT. Rev Ciências de saúde [Internet]. 2016 [acesso em 25 set 2020]. Disponível em: https://repositorio.unb.br/bitstream/10482/20070/I/2015_\%20EdsonOliveiraPereira.pdf.

25 - Ministério da Saúde (BR). Atenção Especializada. Brasília(DF); 2017 [acesso em 03 set 2020]. Disponível em: https://www.saude.gov.br/sistema-unico-de-saude/estrutura-do-sus/770-sistema-nacional-de-saude/403 I7-atencao-especializada.

26 - Borges, S. O Brasil é o país que mais mata LGBT. Clicma [Internet]. 2020 [acesso em 21 agost 2020]. Disponível em: https:// www.cliccamaqua.com.br/noticia/55696/o-brasil-e-o-pais-que -mais-mata-lgbt-afirma-presidente-do-grupo-alianca.html.

27 - Ministério da Saúde (BR). Secretaria de gestão estratégica e participativa. Departamento de apoio á gestão participativa. Transexualidade e travestilidade na saúde. Brasília(DF); 2015. [acesso em 02 set 2020]. Disponível em: http://bvsms. saude.gov.br/bvs/publicacoes/transexualidade_travestilidade_saude.pdf.

28 - Ministério da Saúde (BR). Secretaria de Vigilância em Saúde. Departamento deVigilância, Prevenção e Controle das Infecções Sexualmente Transmissíveis . Prevenção Combinada do HIV: Bases conceituais para profissionais trabalhadores(as) e gestores(as) de saúde. Brasília (DF); 2017. [acesso em 02 set 2020]. Disponível em: http://www.aids.gov.br/pt-br/ pub/20I7/prevencao-combinada-do-hiv-bases-conceituais -para-profissionais-trabalhadoresas-e-gestores.

29 - Oliveira,S.R.; Santos, M.S.; Santos, R.S.; Correira, S.A. O Enfermeiro de Saúde da Familia e Cuidado à População LGBT. Rev International Nursing Congress [Internet]. 2017 [acesso em 02 set 2020]. Disponivel em: https://eventos.set.edu. br/index.php/cie/article/viewFile/5722/2298.

30 - Araújo ET, Sousa G, Carvalho Júnior JA, Pessôa FG de, Moura LK. Acolhimento à população de lésbicas, gays, bissexuais e transgêneros na atenção básica. REAID [Internet]. 30jun.2020 [citado 25set.2020]; 92(30). Available from: https://revistaenfermagematual.com.br/index.php/revista/article/view/637

Recebido: 2020-09-22

Aceito: 2020-10-08 